Applied Mathematical Sciences, Vol. 7, 2013, no. 58, 2841 - 2861

HIKARI Ltd, www.m-hikari.com

\title{
Minimality Concepts Using a New Parameterized Binary Relation in Vector Optimization ${ }^{1}$
}

\author{
Christian Sommer \\ Department Mathematik, Angewandte Mathematik 2 \\ Universität Erlangen-Nürnberg, 91058 Erlangen, Germany \\ sommer@math.fau.de
}

Copyright (c) 2013 Christian Sommer. This is an open access article distributed under the Creative Commons Attribution License, which permits unrestricted use, distribution, and reproduction in any medium, provided the original work is properly cited.

\begin{abstract}
A new parameterized binary relation is used to define minimality concepts in vector optimization. To simplify the problem of determining minimal elements the method of scalarization is applied. Necessary and sufficient conditions for the existence of minimal elements with respect to the scalarized problems are given. The multiplier rule of Lagrange is generalized. As a necessary minimality condition a Karush-KuhnTucker-condition is obtained. The results are applied to optimization problems in finite-dimensional vector spaces.
\end{abstract}

Mathematics Subject Classification: 47N10, 49N10, 65K10

Keywords: Minimality concepts, scalarization, Lagrange multiplier rule, Karush-Kuhn-Tucker-condition

\section{Introduction}

To compare elements of a real Hilbert space $Y$, in a recent paper [13] we have introduced a subset $C_{\varphi} \subset Y$ and a new parameterized binary relation $\preceq_{\varphi}$ (see also [12]). We have defined $C_{\varphi}$ respectively $\preceq_{\varphi}$ by a map $\varphi$ depending on linear operators $A$ and $B$ and a vector $a \in Y$. Relating to these parameters we have

\footnotetext{
${ }^{1}$ This paper contains the second part of the dissertation of the author written at the

University of Erlangen-Nürnberg, 2012
} 
investigated the geometrical and topological properties of $C_{\varphi}$, including the questions when $C_{\varphi} \subsetneq Y, C_{\varphi}$ is bounded, convex or closed and so on.

In this paper we are particularly interested in the properties of the binary relation $\preceq_{\varphi}$. Using it, in Section 2 we define some new minimality concepts like strong, weak and proper minimality. Since it is often difficult to establish the existence of optimal elements in vector optimization, the given problem should be replaced by a suitable scalarized optimization problem with a real valued objective function (see Jahn [6], [7], [9]). The solution of the transformed problem can be easier determined in general. Using the methods of scalarization, in Section 3 we obtain necessary and sufficient conditions for minimal, strongly, properly and weakly minimal elements. In Section 4 we generalize the multiplier rule of Lagrange for our minimality concept and obtain, as a necessary condition for minimality, a Karush-Kuhn-Tucker-condition. Finally we apply the Lagrange multiplier rule to special optimization problems in $Y=\mathbb{R}^{n}$ and $Y=S^{n}$.

\section{Properties of $\preceq_{\varphi}$ and Some Definitions of Minimality}

To define the binary relation $\preceq_{\varphi}$ we follow the lines in [12], [13]: Let $Y$ denote a real Hilbert space with the well-known inner product $\langle\cdot, \cdot\rangle: Y \times Y \rightarrow \mathbb{R}$ and the induced norm $\|x\|:=\sqrt{\langle x, x\rangle}$. Moreover, assume that $Y$ is partially ordered by a convex cone $C_{Y} \subset Y$. Let two linear, continuous and self-adjoint operators $A, B: Y \rightarrow Y$ and a vector $a \in Y$ be given. We define the map $\varphi: Y \times Y \rightarrow \mathbb{R}$ and the subset $C_{\varphi}$ of $Y$ by

$$
\varphi(x, y):=\varphi_{A, B, a}(x, y):=\langle x, A x\rangle-\langle y, B y\rangle+\langle a, x-y\rangle
$$

and

$$
C_{\varphi}:=\{z \in Y: \exists x, y \in Y: z=y-x, \varphi(x, y) \leq 0\} .
$$

Furthermore, for $x, y \in Y$ we introduce the parameterized binary relation which plays the central role in this paper.

$$
x \preceq_{\varphi} y: \Longleftrightarrow y-x \in C_{Y} \text { or, if not, } y-x \in C_{\varphi} \text {. }
$$

To obtain best possible results we make the following convention.

Let $A$ be positive semidefinite and $B$ be negative semidefinite.

Remark 2.1 The additional hypotheses on $A$ and $B$ imply that $\langle x, A x\rangle \geq 0$ and $\langle y, B y\rangle \leq 0$ for all $x, y \in Y$. We have shown in [12], [13] that then $\operatorname{int}\left(C_{\varphi}\right) \neq \emptyset, C_{\varphi}$ is convex and contained in the half space $H^{+}:=\{z \in Y$ : 
$\langle a, z\rangle \geq 0\}$. Moreover, we have determined the dual set $C_{\varphi}^{*}$ of $C_{\varphi}$ and the contingent cone at an arbitrary $\bar{z} \in C_{\varphi} \cap H$ where $H:=\{z \in Y:\langle a, z\rangle=0\}$. Thus, it follows that $C_{\varphi}$ has the most substantial mathematical structure under our convention.

Analogously to $C_{\varphi}$ we define the set $D_{\varphi}$ by

$$
D_{\varphi}:=\left\{z \in Y: \exists x, y \in Y: z=y-x, x \preceq_{\varphi} y\right\} .
$$

Remark 2.2 (i) It is easily seen that

$$
D_{\varphi}=C_{Y} \cup C_{\varphi}
$$

(ii) By [12], Th. 1.17 the relation $\preceq_{\varphi}$ represents a partial ordering iff $D_{\varphi}$ is a convex cone.

(iii) An example for a convex cone $D_{\varphi}$ is given by Example 2.100 in [12] $\left(C_{Y}=\mathbb{R}_{+}^{3}\right)$. On the other hand, the Examples 6.1 and 6.2 in $[13]\left(C_{Y}=\mathbb{R}_{+}^{2}\right)$ describe situations where $D_{\varphi}$ is neither convex nor a cone.

Minimal elements are usually defined with respect to a partial ordering, i.e., an ordering cone is needed (see Jahn [9], pp. 103). Since in our situation $D_{\varphi}$ could fail to be a cone, we here present minimal concepts in a more general way. To obtain reasonable connections between our different concepts we assume the property

$$
C_{Y} \subset H^{+} \text {. }
$$

Since by (2.4) the inclusion $C_{\varphi} \subset H^{+}$holds, we are then actually concerned with the situation that $D_{\varphi} \subset H^{+}$. For the general case (without assuming (2.6)) we refer to Weidner [16]. In that paper several minimality concepts have been defined with respect to general subsets $D$ of $Y$ with the only property that $D \backslash\left\{0_{Y}\right\} \neq \emptyset$.

Let us now assume that (2.6) is always satisfied in the following.

Definition 2.3 Let $T \subset Y, T \neq \emptyset$. An $\bar{x} \in T$ is called a $D_{\varphi}$-minimal element of $T$ if

$$
\left(\{\bar{x}\}-D_{\varphi}\right) \cap T \subset\{\bar{x}\}+D_{\varphi} .
$$

The following characterization of $D_{\varphi}$-minimal elements can be easily verified (see [12], Lemma 3.3).

Lemma 2.4 Let $T \subset Y, T \neq \emptyset$ and $\bar{x} \in T$. The following statements are equivalent.

(a) $\bar{x}$ is a $D_{\varphi}$-minimal element of $T$. 
(b) There is no $x \in T$ such that $\bar{x} \in\{x\}+D_{\varphi} \backslash\left(-D_{\varphi}\right)$.

Remark 2.5 Weidner [16], pp. 89 defines so-called efficient elements according to Lemma 2.4, (b) as a criterion for optimality.

The simple proof of the following lemma is given in [12], Lemma 3.6.

Lemma 2.6 If $D_{\varphi}$ is pseudo-pointed, i.e., $D_{\varphi} \cap H=\left\{0_{Y}\right\}$, property (2.7) can be replaced by

$$
\left(\{\bar{x}\}-D_{\varphi}\right) \cap T=\{\bar{x}\} .
$$

Of course, a minimal (or more general an optimal) element could be interpreted as a lower bound of a given set. This leads to the following notation.

Definition 2.7 Let $T \subset Y, T \neq \emptyset$. An $\bar{x} \in T$ is called a strongly $D_{\varphi^{-}}$ minimal element of $T$ if

$$
T \subset\{\bar{x}\}+D_{\varphi}
$$

Remark 2.8 It is obvious that an $\bar{x} \in T$ is a strongly $D_{\varphi}$-minimal element of $T$ iff $x-\bar{x} \in D_{\varphi}$ holds for all $x \in T$.

The following statement is an easy consequence of the above definitions.

Lemma 2.9 Let $T \subset Y, T \neq \emptyset$. Then, every strongly $D_{\varphi}$-minimal element of $T$ is a $D_{\varphi}$-minimal element of $T$.

To define the next minimality notion we need the concept of a contingent cone.

Definition 2.10 Let $(X,\|\cdot\|)$ be a real normed space and $S \subset X, S \neq \emptyset$.

(a) Let some $\bar{x} \in \operatorname{cl}(S)$ (the closure of $S$ ) be given. An element $h \in X$ is called a tangent to $S$ at $\bar{x}$, if there are a sequence $\left(x_{n}\right)_{n \in \mathbb{N}} \subset S$ and a sequence $\left(\lambda_{n}\right)_{n \in \mathbb{N}}$ of positive real numbers such that

$$
\bar{x}=\lim _{n \rightarrow \infty} x_{n} \quad \text { and } \quad h=\lim _{n \rightarrow \infty} \lambda_{n}\left(x_{n}-\bar{x}\right) .
$$

(b) The set $\mathcal{T}(S, \bar{x})$ of all tangents to $S$ at $\bar{x}$ is called contingent cone to $S$ at $\bar{x}$.

Remark 2.11 Vogel [14], p. 78 uses contingent cones to define the properties local-efficient, local Bayes and properly efficient. Moreover, proper minimality (defined by contingent cones) plays an important role in the research of Borwein ([1], [2]). In this context we introduce proper $D_{\varphi}$-minimality as follows. 
Definition 2.12 Let $T \subset Y, T \neq \emptyset$. An $\bar{x} \in T$ is called a properly $D_{\varphi^{-}}$ minimal element of $T$ if $\bar{x}$ is a $D_{\varphi}$-minimal element of $T$ and $0_{Y}$ is a $D_{\varphi}$-minimal element of the contingent cone $\mathcal{T}\left(T+D_{\varphi}, \bar{x}\right)$.

Finally, we discuss a weaker property than the above defined minimality concepts. For this purpose let us observe that $\operatorname{int}\left(D_{\varphi}\right) \neq \emptyset$ by Remark 2.1.

Definition 2.13 Let $T \subset Y, T \neq \emptyset$. An $\bar{x} \in T$ is called a weakly $D_{\varphi^{-}}$ minimal element of $T$ if

$$
\left(\{\bar{x}\}-\operatorname{int}\left(D_{\varphi}\right)\right) \cap T=\emptyset .
$$

The following lemma shows a connection between the concepts of $D_{\varphi}$-minimality and weak $D_{\varphi}$-minimality (see [12], Lemma 3.15 for the simple proof).

Lemma 2.14 Let $T \subset Y, T \neq \emptyset$. Then, every $D_{\varphi}$-minimal element of $T$ is a weakly $D_{\varphi}$-minimal element of $T$.

The next result presents a useful property of weakly $D_{\varphi}$-minimal elements (see [12], Lemma 3.16 for the simple proof).

Lemma 2.15 Let $T \subset Y, T \neq \emptyset$. Then, every weakly $D_{\varphi}$-minimal element $\bar{x} \in T$ of the set $T+D_{\varphi}$ is a weakly $D_{\varphi}$-minimal element of $T$.

The converse of Lemma 2.15 is not true in general. Let us consider the following example.

Example 2.16 Let $Y=\mathbb{R}^{2}$. We define $A, B$ and vectors $a, v, \bar{x} \in \mathbb{R}^{2}$ by

$$
A:=\left(\begin{array}{ll}
1 & 0 \\
0 & 0
\end{array}\right), B:=\left(\begin{array}{cc}
-1 & 0 \\
0 & 0
\end{array}\right), a:=\left(\begin{array}{l}
1 \\
1
\end{array}\right), v:=\left(\begin{array}{c}
-1 \\
0
\end{array}\right), \bar{x}:=0_{\mathbb{R}^{2}} .
$$

As we have shown in [12], Ex. 3.8, $C_{\varphi}$ satisfies the equality

$$
C_{\varphi}=\left\{z=\left(z_{1}, z_{2}\right)^{T} \in \mathbb{R}^{2}: z_{2} \geq \frac{1}{2} z_{1}^{2}-z_{1}\right\}
$$

For $C_{Y}=\mathbb{R}_{+}^{2}$ we then obtain

$$
D_{\varphi}=\mathbb{R}_{+}^{2} \cup\left\{z=\left(z_{1}, z_{2}\right)^{T} \in \mathbb{R}^{2}: z_{2} \geq \frac{1}{2} z_{1}^{2}-z_{1}\right\} .
$$

Let us consider the set $T:=\left(\mathbb{R}^{2} \backslash\left(-D_{\varphi}\right)\right) \cup\left\{0_{\mathbb{R}^{2}}\right\}$. Then, it is easily seen that $\bar{x}$ is a $D_{\varphi}$-minimal element of $T$ and, in view of Lemma 2.14, also a weakly 
$D_{\varphi}$-minimal element of $T$. To argue that the converse of Lemma 2.15 is not true we observe that

$$
v=(-1,0)^{T}=\left(-2, \frac{1}{2}\right)^{T}+\left(1,-\frac{1}{2}\right)^{T} \in T+C_{\varphi} \subset T+D_{\varphi}
$$

and

$$
-v=(1,0)^{T} \in \operatorname{int}\left(C_{\varphi}\right) \subset \operatorname{int}\left(D_{\varphi}\right) .
$$

Hence, we obtain $v \in\left(\{\bar{x}\}-\operatorname{int}\left(D_{\varphi}\right)\right) \cap\left(T+D_{\varphi}\right)$ which clearly implies that

$$
\left(\{\bar{x}\}-\operatorname{int}\left(D_{\varphi}\right)\right) \cap\left(T+D_{\varphi}\right) \supsetneq\{\bar{x}\} .
$$

Thus, it follows that $\bar{x}$ fails to be a weakly $D_{\varphi}$-minimal element of $T+D_{\varphi}$.

\section{Scalarization}

As we have already explained in the introduction, a given problem in vector optimization can sometimes be better handled after replacing it by a suitable scalarized optimization problem with a real valued objective function. Since the theory of scalarized optimization is largely studied, the solution of the transformed problem can be easier determined in general. Mainly two methods of scalarization are applied, by using linear or nonlinear functionals respectively norms or semi-norms (see [6], [7], [9]). In [3] - [5] nonlinear functionals are constructed to separate nonconvex sets. The properties of such functionals are discussed in detail in [17], [18]. Moreover, in [5] results on scalarization are obtained for the case of weakly and properly efficient elements which have been first defined and studied in [15] and [16].

Let us again assume that $D_{\varphi}$ is given as in Section 2, i.e., $D_{\varphi} \subset H^{+}$. Applying the above mentioned methods of scalarization, in the following subsections we derive some necessary and sufficient conditions for $D_{\varphi}$-minimal, properly $D_{\varphi}$-minimal and weakly $D_{\varphi}$-minimal elements of a set $T$. For our studies we can come back to Definition 2.12 and Lemma 2.14 observing that every sufficient condition for proper $D_{\varphi}$-minimality is sufficient for $D_{\varphi}$-minimality and also for weak $D_{\varphi}$-minimality. On the other hand, every necessary condition for weak $D_{\varphi}$-minimality is necessary for $D_{\varphi}$-minimality and also for proper $D_{\varphi}$-minimality.

Definition 3.1 Let subsets $T_{1}$ and $T_{2}$ of $Y$ be given such that $\emptyset \neq T_{1} \subset T_{2}$.

(a) A functional $f: T_{2} \rightarrow \mathbb{R}$ is called monotonically increasing (resp. strongly monotonically increasing) on $T_{1}$, if for every $\bar{x} \in T_{1}$ the condition

$$
\left.x \in\left(\{\bar{x}\}-D_{\varphi}\right) \cap T_{1}, x \neq \bar{x} \Longrightarrow f(x) \leq f(\bar{x}) \quad \text { (resp. } f(x)<f(\bar{x})\right)
$$

holds. 
(b) A functional $f: T_{2} \rightarrow \mathbb{R}$ is called strictly monotonically increasing on $T_{1}$, if for every $\bar{x} \in T_{1}$ the condition

$$
x \in\left(\{\bar{x}\}-\operatorname{int}\left(D_{\varphi}\right)\right) \cap T_{1} \Longrightarrow f(x)<f(\bar{x})
$$

holds.

Remark 3.2 (i) Of course, every strongly monotonically increasing functional on $T_{1}$ is also strictly monotonically increasing.

(ii) It is well-known that a linear functional $l: Y \rightarrow \mathbb{R}$ on a real Hilbert space $(Y,\langle\cdot, \cdot\rangle)$ is given by a scalar product $\langle v, \cdot\rangle$ with $v=v(l) \in Y$. Since this special representation is irrelevant in the following, we use the general notion $l$.

\subsection{Scalarization concerning $D_{\varphi}$-minimality}

Let us begin our studies on scalarization with the property of $D_{\varphi}$-minimality.

Theorem 3.3 Let $T \subset Y, T \neq \emptyset$ and assume that the set $T+D_{\varphi}$ is convex. Moreover, assume for some $\bar{x} \in T$ that $\bar{x}$ is both a $D_{\varphi}$-minimal element of the set $T$ and a $D_{\varphi}$-minimal element of the set $T+D_{\varphi}$. Then, there is a linear functional $l \in Y^{\prime} \backslash\left\{0_{Y^{\prime}}\right\}$ such that

$$
l(\bar{x}) \leq l(x) \text { for all } x \in T
$$

$\left(Y^{\prime}\right.$ denotes the algebraic dual space of $\left.Y\right)$.

This theorem has a generalized analogue in Section 3.3, namely Theorem 3.14. Hence, the statement follows immediately from that result.

Remark 3.4 As we have shown by Example 2.16, a $D_{\varphi}$-minimal element $\bar{x} \in T$ of $T$ is not necessarily a $D_{\varphi}$-minimal element of $T+D_{\varphi}$. Hence, such a property has to be additionally assumed in Theorem 3.3. However, this assumption becomes redundant if the minimality is defined by an ordering cone $C_{Y}$ (see [9], Th. 5.4).

We are now in position to establish a characterization of $D_{\varphi}$-minimality by using linear functionals.

Theorem 3.5 Let $T \subset Y, T \neq \emptyset$ and assume that $D_{\varphi}$ is closed, convex and pseudo-pointed. Then, an element $\bar{x} \in T$ is a $D_{\varphi}$-minimal element of $T$ iff for every $x \in T \backslash\{\bar{x}\}$ there are a continuous linear functional $l \in Y^{*} \backslash\left\{0_{Y^{*}}\right\}$ and an $\alpha \in \mathbb{R}$ such that

$$
l(\bar{x}-d) \leq \alpha<l(x) \text { for all } d \in D_{\varphi} .
$$

If this is the case, then

$$
l(\bar{x})<l(x) .
$$


Proof: Let $\bar{x} \in T$ be a $D_{\varphi}$-minimal element of $T$. Since $D_{\varphi}$ is pseudopointed, by Lemma 2.6 this means

$$
\left(\{\bar{x}\}-D_{\varphi}\right) \cap T=\{\bar{x}\} .
$$

This property is equivalent to

$$
x \notin\{\bar{x}\}-D_{\varphi} \text { for all } x \in T \backslash\{\bar{x}\} .
$$

Since $D_{\varphi}$ is nonempty, convex and closed, the set $\{\bar{x}\}-D_{\varphi}$ has the same properties. Hence, we can apply a well-known separation theorem (see [9], pp. 75): (3.5) is then equivalent to the statement that for every $x \in T \backslash\{\bar{x}\}$ there are a continuous linear functional $\bar{l} \in Y^{*} \backslash\left\{0_{Y^{*}}\right\}$ and an $\bar{\alpha} \in \mathbb{R}$ such that

$$
\bar{l}(x)<\bar{\alpha} \leq \bar{l}(\bar{x}-d) \text { for all } d \in D_{\varphi} .
$$

With $l:=-\bar{l}$ and $\alpha:=-\bar{\alpha}$ (3.6) is equivalent to assertion (3.2). Moreover, inequality (3.3) follows immediately from (3.2) setting $d=0_{Y} \in D_{\varphi}$.

We are now able to present another necessary and sufficient condition for $D_{\varphi}$-minimality where the normal vector $a$ of $H$ plays an important role. The rather elementary proof can be found in [12], Th. 4.7.

Theorem 3.6 Let $T \subset Y, T \neq \emptyset$ and $\bar{x} \in T$. Assume that $D_{\varphi}$ is pseudopointed. Then the following statements hold.

(a) If the property $\langle a, \bar{x}\rangle \leq\langle a, x\rangle$ is satisfied for all $x \in T$, then $\bar{x}$ is a $D_{\varphi}$-minimal element of $T$.

(b) If $T$ is convex, then the converse of statement (a) is also true.

The hypothesis on $T$ to be convex is essential in statement (b) as the following example shows.

Example 3.7 Let $Y=\mathbb{R}^{2}$. We define $A, B$, the nonconvex set $T$ and vectors $a, \bar{x} \in \mathbb{R}^{2}$ as in Example 2.16. Then, $D_{\varphi}$ is pseudo-pointed and $\bar{x}=0_{\mathbb{R}^{2}}$ is a $D_{\varphi}$-minimal element of $T$. On the other hand, setting $v:=(-2,1)^{T} \in T$ we obtain $a^{T} v=(1,1)(-2,1)^{T}=-1<0=a^{T} \bar{x}$. Hence, the converse of Theorem 3.6 (a) does not hold.

Using the induced norm on $Y$ we give a sufficient condition for $D_{\varphi}$-minimality.

Theorem 3.8 Let $T \subset Y, T \neq \emptyset$. Moreover, let $\hat{x} \in Y$ and $\bar{x} \in T$ be given such that

$$
T \subset\{\hat{x}\}+D_{\varphi}
$$

and

$$
\|\bar{x}-\hat{x}\| \leq\|x-\hat{x}\| \text { for all } x \in T .
$$

Then the following statements hold. 
(a) If the norm $\|\cdot\|$ is monotonically increasing on $D_{\varphi}$ and $\bar{x}$ is uniquely determined by (3.8), then $\bar{x}$ is a $D_{\varphi}$-minimal element of $T$.

(b) If $\|\cdot\|$ is strongly monotonically increasing on $D_{\varphi}$, then $\bar{x}$ is a $D_{\varphi}$-minimal element of $T$.

To prove the statement we need a lemma (see [9], pp. 129 for the proof).

Lemma 3.9 Let $T \subset Y, T \neq \emptyset$. Moreover, let a functional $f: T \rightarrow \mathbb{R}$ and an $\bar{x} \in T$ be given such that

$$
f(\bar{x}) \leq f(x) \text { for all } x \in T \text {. }
$$

Then the following statements hold.

(a) If $f$ is monotonically increasing on $T$ and $\bar{x}$ is uniquely determined by (3.9), then $\bar{x}$ is a $D_{\varphi}$-minimal element of $T$.

(b) If $f$ is strongly monotonically increasing on $T$, then $\bar{x}$ is a $D_{\varphi}$-minimal element of $T$.

(c) If $f$ is strictly monotonically increasing on $T$, then $\bar{x}$ is a weakly $D_{\varphi^{-}}$ minimal element of $T$.

Proof of Theorem 3.8: We prove only statement (a); the proof of (b) follows analogously. For this purpose we follow the arguments in [9], p. 131, but without the concept of an ordering interval. Of course, we can apply Lemma 3.9 (a) after having shown that the functional

$$
f: T \rightarrow \mathbb{R}, \quad f(x):=\|x-\hat{x}\|
$$

is monotonically increasing on $T$. Let $\bar{t} \in T$ be given. We have to show that $f(x) \leq f(\bar{t})$ holds for every $x \in\left(\{\bar{t}\}-D_{\varphi}\right) \cap T$. By (3.7) we obtain $\bar{t}-\hat{x} \in D_{\varphi}$ and

$$
\left(\{\bar{t}\}-D_{\varphi}\right) \cap T \subset\left(\{\bar{t}\}-D_{\varphi}\right) \cap\left(\{\hat{x}\}+D_{\varphi}\right) .
$$

Moreover, using that $x \in\left(\{\bar{t}\}-D_{\varphi}\right) \cap T$ and (3.10) we obtain $(\bar{t}-\hat{x})-(x-\hat{x})=$ $\bar{t}-x \in D_{\varphi}$ and $x \in\{\hat{x}\}+D_{\varphi}$. This implies $x-\hat{x} \in\left(\{\bar{t}-\hat{x}\}-D_{\varphi}\right) \cap D_{\varphi}$. Since by assumption $\|\cdot\|$ is monotonically increasing on $D_{\varphi}$, the relation

$$
f(x)=\|x-\hat{x}\| \leq\|\bar{t}-\hat{x}\|=f(\bar{t})
$$

follows. Hence, $f$ is monotonically increasing on $T$. Since by assumption $\bar{x}$ is uniquely determined by (3.8), it is a $D_{\varphi}$-minimal element of $T$ by Lemma 3.9 (a). 
Remark 3.10 Property (3.7) obviously means that $\hat{x}$ can be considered as a lower bound of $T$. But also for the case when $T$ does not satisfy such a property, approximation problems are suitable for determining $D_{\varphi}$-minimal elements of $T$. This is, however, only possible under additional hypotheses on $D_{\varphi}$ as the following result shows (see [12], Th. 4.11 for the proof).

Theorem 3.11 Let $T \subset Y, T \neq \emptyset$. Moreover, assume that $D_{\varphi}$ satisfies the property $D_{\varphi}+D_{\varphi} \subset D_{\varphi}$. Let $\tilde{x} \in T$ and $\bar{x} \in T \cap\left(\{\tilde{x}\}-D_{\varphi}\right)$ be given such that

$$
\|\bar{x}-\tilde{x}\| \geq\|x-\tilde{x}\| \text { for all } x \in T \cap\left(\{\tilde{x}\}-D_{\varphi}\right) .
$$

(a) If the norm $\|\cdot\|$ is monotonically increasing on $D_{\varphi}$ and $\bar{x}$ is uniquely determined by (3.11), then $\bar{x}$ is a $D_{\varphi}$-minimal element of $T$.

(b) If $\|\cdot\|$ is strongly monotonically increasing on $D_{\varphi}$, then $\bar{x}$ is a $D_{\varphi}$-minimal element of $T$.

Remark 3.12 (i) We have shown in [12], Lemma 4.12 that the property $D_{\varphi}+D_{\varphi} \subset D_{\varphi}$ implies that $D_{\varphi}$ is a convex cone. This strong property is also needed in the Theorems 3.13 and 3.18 below.

(ii) In general, the set $D_{\varphi}$ fails to satisfy $D_{\varphi}+D_{\varphi} \subset D_{\varphi}$.

(iii) In contrast to Theorem 3.8 where the minimal distance between $\hat{x}$ and $T$ is essential, in Theorem 3.11 the maximal distance between $\tilde{x}$ and $T \cap\left(\{\tilde{x}\}-D_{\varphi}\right)$ plays a central role.

\subsection{Scalarization concerning proper $D_{\varphi}$-minimality}

We give here a sufficient condition for proper $D_{\varphi}$-minimality under a strong additional hypothesis on $D_{\varphi}$.

Theorem 3.13 Let $T \subset Y, T \neq \emptyset$. Moreover, assume that $D_{\varphi}$ satisfies the property $D_{\varphi}+D_{\varphi} \subset D_{\varphi}$. Let an $\hat{x} \in Y$ be given such that $T \subset\{\hat{x}\}+\operatorname{int}\left(D_{\varphi}\right)$. If the norm $\|\cdot\|$ is strongly monotonically increasing on $D_{\varphi}$ and if there is an $\bar{x} \in T$ such that

$$
\|\bar{x}-\hat{x}\| \leq\|x-\hat{x}\| \text { for all } x \in T,
$$

then $\bar{x}$ is a properly $D_{\varphi}$-minimal element of $T$.

Proof: We follow the arguments in [9], p. 134. But in contrast to that, our set $D_{\varphi}$ is not assumed to be pseudo-pointed. Therefore, we have to make slight changes within the proof.

Since $\|\cdot\|$ is strongly monotonically increasing on $D_{\varphi}$ and, therefore, on $T-\{\hat{x}\} \subset \operatorname{int}\left(D_{\varphi}\right) \subset D_{\varphi}$, the functional

$$
f: T \rightarrow \mathbb{R}, \quad f(x):=\|x-\hat{x}\|
$$


is strongly monotonically increasing on $T$. Hence, by Lemma $3.9(\mathrm{~b}), \bar{x}$ is a $D_{\varphi}$-minimal element of $T$.

Next we want to show that $0_{Y}$ is a $D_{\varphi}$-minimal element of the contingent cone $\mathcal{T}\left(T+D_{\varphi}, \bar{x}\right)$. Since $\bar{x} \in T, T-\{\hat{x}\} \subset D_{\varphi}$ and $D_{\varphi}+D_{\varphi} \subset D_{\varphi}$, for all $x \in T$ and $d \in D_{\varphi}$ we obtain

$$
x-\hat{x}+d \in T-\{\hat{x}\}+D_{\varphi} \subset D_{\varphi}+D_{\varphi} \subset D_{\varphi}
$$

and

$$
x-\hat{x} \in\left(\{x-\hat{x}+d\}-D_{\varphi}\right) \cap D_{\varphi} .
$$

Moreover, since $\|\cdot\|$ is strongly monotonically increasing on $D_{\varphi}$, from (3.12) - (3.14) we conclude

$$
\|\bar{x}-\hat{x}\| \leq\|x-\hat{x}\| \leq\|x+d-\hat{x}\| \text { for all } x \in T, d \in D_{\varphi} .
$$

This implies

$$
\|\bar{x}-\hat{x}\| \leq\|x-\hat{x}\| \text { for all } x \in T+D_{\varphi} .
$$

It is easily seen that $f$ is continuous and convex. Then, using (3.15) and a known result on contingent cones (see [9], pp. 94) we obtain the inequality

$$
\|\bar{x}-\hat{x}\| \leq\|\bar{x}-\hat{x}+h\| \text { for all } h \in \mathcal{T}\left(T+D_{\varphi}, \bar{x}\right) .
$$

Define the set $\tilde{T}$ and the functional $g$ by $\tilde{T}:=\mathcal{T}\left(T+D_{\varphi}, \bar{x}\right) \cap\left(\{\hat{x}-\bar{x}\}+D_{\varphi}\right)$ and

$$
g: \tilde{T} \rightarrow \mathbb{R}, \quad g(h):=\|\bar{x}-\hat{x}+h\| .
$$

Since $\tilde{T} \subset \mathcal{T}\left(T+D_{\varphi}, \bar{x}\right)$, by (3.16) we obtain

$$
g\left(0_{Y}\right)=\|\bar{x}-\hat{x}\| \leq\|\bar{x}-\hat{x}+h\|=g(h) \text { for all } h \in \tilde{T} .
$$

Moreover, since $\|\cdot\|$ is strongly monotonically increasing on $D_{\varphi}, g$ has the same property on $\left(\{\hat{x}-\bar{x}\}+D_{\varphi}\right) \cap \tilde{T}=\tilde{T}$. Hence, by Lemma 3.9 (b), $0_{Y}$ is a $D_{\varphi}$-minimal element of $\tilde{T}$.

To complete the proof let us assume that $0_{Y}$ fails to be a $D_{\varphi}$-minimal element of the contingent cone $\mathcal{T}\left(T+D_{\varphi}, \bar{x}\right)$. Then, there is an element $x \in$ $\left(\left\{0_{Y}\right\}-D_{\varphi}\right) \cap \mathcal{T}\left(T+D_{\varphi}, \bar{x}\right)$ such that $x \notin\left\{0_{Y}\right\}+D_{\varphi}$. Since $\bar{x} \in T$ and $T \subset\{\hat{x}\}+\operatorname{int}\left(D_{\varphi}\right)$, the property $\bar{x}-\hat{x} \in \operatorname{int}\left(D_{\varphi}\right)$ holds. Hence, there is a $\lambda>0$ such that $\bar{x}-\hat{x}+\lambda x \in D_{\varphi}$ resp. $\lambda x \in\{\hat{x}-\bar{x}\}+D_{\varphi}$. Then, since $\mathcal{T}\left(T+D_{\varphi}, \bar{x}\right)$ and $-D_{\varphi}$ (by Remark 3.12) are cones, we obtain

$$
\lambda x \in\left(-D_{\varphi}\right) \cap \mathcal{T}\left(T+D_{\varphi}, \bar{x}\right) \cap\left(\{\hat{x}-\bar{x}\}+D_{\varphi}\right)=\left(-D_{\varphi}\right) \cap \tilde{T} .
$$

Moreover, $\lambda x \notin D_{\varphi}$, because $x \notin D_{\varphi}$. Altogether, the inclusion

$$
\left(\left\{0_{Y}\right\}-D_{\varphi}\right) \cap \tilde{T} \subset\left\{0_{Y}\right\}+D_{\varphi}
$$

is violated, a contradiction to the property that $0_{Y}$ is a $D_{\varphi}$-minimal element of $\tilde{T}$. This completes the proof. 


\subsection{Scalarization concerning weak $D_{\varphi}$-minimality}

First we give a necessary condition for weak $D_{\varphi}$-minimality generalizing Theorem 3.3.

Theorem 3.14 Let $T \subset Y, T \neq \emptyset$ and assume that the set $T+D_{\varphi}$ is convex. Moreover, assume for some $\bar{x} \in T$ that $\bar{x}$ is both a weakly $D_{\varphi}$-minimal element of the set $T$ and a weakly $D_{\varphi}$-minimal element of the set $T+D_{\varphi}$. Then, there is a linear functional $l \in Y^{\prime} \backslash\left\{0_{Y^{\prime}}\right\}$ such that

$$
l(\bar{x}) \leq l(x) \text { for all } x \in T .
$$

Proof: Since $\bar{x}$ is a weakly $D_{\varphi}$-minimal element of $T+D_{\varphi}$, by Definition 2.13 we obtain

$$
\left(\{\bar{x}\}-\operatorname{int}\left(D_{\varphi}\right)\right) \cap\left(T+D_{\varphi}\right)=\emptyset .
$$

Let an arbitrary $t \in T$ be given. It is then easily seen that

$$
\operatorname{int}\left(T+D_{\varphi}\right) \supset \operatorname{int}\left(\{t\}+D_{\varphi}\right)=\{t\}+\operatorname{int}\left(D_{\varphi}\right) \neq \emptyset .
$$

We want to show that $\operatorname{int}\left(T+D_{\varphi}\right) \cap\{\bar{x}\}=\emptyset$. On the contrary suppose that $\bar{x} \in \operatorname{int}\left(T+D_{\varphi}\right)$. Hence, there is an $\epsilon>0$ such that $B_{\epsilon}(\bar{x})=\{v \in Y$ : $\|v-\bar{x}\|<\epsilon\} \subset T+D_{\varphi}$. Then, using Remark 2.2 und (3.19) we obtain

$$
\left(\{\bar{x}\}-\operatorname{int}\left(C_{\varphi}\right)\right) \cap B_{\epsilon}(\bar{x}) \subset\left(\{\bar{x}\}-\operatorname{int}\left(D_{\varphi}\right)\right) \cap\left(T+D_{\varphi}\right)=\emptyset .
$$

Let $z \in H^{+} \backslash H$ and let $\lambda>0$ be sufficiently small such that $\lambda<\frac{\epsilon}{\|z\|}$ and

$$
\lambda\langle z, B z\rangle+\langle a, z\rangle>0 .
$$

We define $w \in Y$ by $w:=\bar{x}-\lambda z$. Using (3.21) we obtain

$$
\begin{aligned}
\varphi\left(0_{Y}, \lambda z\right) & =-\langle\lambda z, B(\lambda z)\rangle+\left\langle a, 0_{Y}-\lambda z\right\rangle \\
& =-\lambda^{2}\langle z, B z\rangle-\lambda\langle a, z\rangle=-\lambda(\lambda\langle z, B z\rangle+\langle a, z\rangle)<0 .
\end{aligned}
$$

This implies $\lambda z=\lambda z-0_{Y} \in \operatorname{int}\left(C_{\varphi}\right)$ (see also [12], Th. 2.37). Thus, it follows $w=\bar{x}-\lambda z \in\{\bar{x}\}-\operatorname{int}\left(C_{\varphi}\right)$ and $\|w-\bar{x}\|=\|-\lambda z\|=\lambda\|z\|<\epsilon$, i.e., $w \in B_{\epsilon}(\bar{x})$, a contradiction to $(3.20)$.

Since $\operatorname{int}\left(T+D_{\varphi}\right) \cap\{\bar{x}\}=\emptyset$, we can apply a well-known separation theorem (see [9], pp. 72) to the convex sets $T+D_{\varphi}$ and $\{\bar{x}\}$. Hence, there are a linear functional $\bar{l} \in Y^{\prime} \backslash\left\{0_{Y^{\prime}}\right\}$ and an $\bar{\alpha} \in \mathbb{R}$ such that

$$
\bar{l}(t+d) \leq \bar{\alpha} \leq \bar{l}(\bar{x}) \text { for all } t \in T, d \in D_{\varphi} .
$$

Setting $l:=-\bar{l}, \alpha:=-\bar{\alpha}$ and $d=0_{Y} \in D_{\varphi}$ in (3.22), we have obtained statement (3.18).

The next statement is similar to Theorem 3.6. The rather elementary proof can be found in [12], Th. 4.16. 
Theorem 3.15 Let $T \subset Y, T \neq \emptyset$ and $\bar{x} \in T$. Then the following statements hold.

(a) If the property $\langle a, \bar{x}\rangle \leq\langle a, x\rangle$ is satisfied for all $x \in T$, then $\bar{x}$ is a weakly $D_{\varphi}$-minimal element of $T$.

(b) If $T$ is convex, then the converse of statement (a) is also true.

Remark 3.16 The hypothesis on $T$ to be convex is essential in statement (b). Let us again consider Example 2.16. As we have shown there the vector $\bar{x}=0_{\mathbb{R}^{2}}$ is a $D_{\varphi}$-minimal and, therefore, a weakly $D_{\varphi}$-minimal element of the nonconvex set $T$. On the other hand, setting $v:=(-2,1)^{T} \in T$ we obtain $a^{T} v=(1,1)(-2,1)^{T}=-1<0=a^{T} \bar{x}$. Hence, the converse of Theorem 3.15 (a) does not hold.

We now give a sufficient condition for weak $D_{\varphi}$-minimality of subsets $T$ which have a lower bound.

Theorem 3.17 Let $T \subset Y, T \neq \emptyset$ and let an $\hat{x} \in Y$ be given such that

$$
T \subset\{\hat{x}\}+D_{\varphi}
$$

If the norm $\|\cdot\|$ is strictly monotonically increasing on $D_{\varphi}$ and if there is an element $\bar{x} \in T$ satisfying

$$
\|\bar{x}-\hat{x}\| \leq\|x-\hat{x}\| \text { for all } x \in T \text {, }
$$

then $\bar{x}$ is a weakly $D_{\varphi}$-minimal element of $T$.

Proof: The statement can be verified by similar arguments as in the proof of Theorem 3.8 .

We finish this section by giving another sufficient condition for weak $D_{\varphi^{-}}$ minimality.

Theorem 3.18 Let $T \subset Y, T \neq \emptyset$ and assume that $D_{\varphi}+D_{\varphi} \subset D_{\varphi}$. Moreover, let an $\tilde{x} \in T$ be given. If the norm $\|\cdot\|$ is strictly monotonically increasing on $D_{\varphi}$ and if there is an element $\bar{x} \in T \cap\left(\{\tilde{x}\}-D_{\varphi}\right)$ such that

$$
\|\bar{x}-\tilde{x}\| \geq\|x-\tilde{x}\| \text { for all } x \in T \cap\left(\{\tilde{x}\}-D_{\varphi}\right),
$$

then $\bar{x}$ is a weakly $D_{\varphi}$-minimal element of $T$.

Proof: The statement can be verified by similar arguments as in the proof of Theorem 3.11 (see also [12], Th. 4.20). 


\section{Karush-Kuhn-Tucker-Condition}

In this section we generalize the multiplier rule of Lagrange for the concept of $D_{\varphi}$-minimality. For this purpose we consider an abstract optimization problem with constraints given by equalities and inequalities. As a necessary condition for minimality we establish a Karush-Kuhn-Tucker-condition (KKT). We restrict ourselves to the concept of weak $D_{\varphi}$-minimality considering the fact

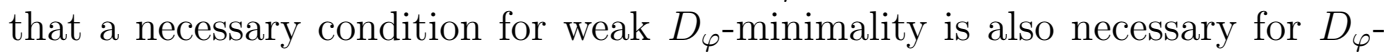
minimality (see Lemma 2.14). Moreover, to obtain such conditions we use Fr?chet differentiable maps. It is also possible to formulate the Lagrange multiplier rule by means of a more general class of differentiable maps (see e.g. [10]). We finally apply our results to the case of finite-dimensional Hilbert spaces, especially studying two concrete optimization problems.

Our studies have been motivated by known results on KKT-conditions which can be found in [8], pp. 187 and [9], pp. 161. More detailed, we are now concerned with the following situation:

Assume that $\left(X,\|\cdot\|_{X}\right)$ and $\left(Z_{2},\|\cdot\|_{Z_{2}}\right)$ are real Banach spaces.

Moreover, let $(Y,\langle\cdot, \cdot\rangle)$ be the given real Hilbert space and

$\left(Z_{1},\|\cdot\|_{Z_{1}}\right)$ be a partial ordered normed space with ordering cone $C_{Z_{1}}$

such that $\operatorname{int}\left(C_{Z_{1}}\right) \neq \emptyset$. Assume that $D_{\varphi}$ is convex and satisfies

$D_{\varphi} \subset H^{+}$. Moreover, let $\hat{S} \subset X$ such that $\operatorname{int}(\hat{S}) \neq \emptyset$ and $\hat{S}$ is convex.

Let maps $f: X \rightarrow Y, g: X \rightarrow Z_{1}$ and $h: X \rightarrow Z_{2}$ be given.

We define the feasible set $S$ by

$$
S:=\left\{x \in \hat{S}: g(x) \in-C_{Z_{1}}, h(x)=0_{Z_{2}}\right\} .
$$

Assuming that $S \neq \emptyset$ we consider the abstract optimization problem

$$
\min _{x \in S} f(x)
$$

The map $f$ is called objective function. This leads to the following definition.

Definition 4.1 Assume that (4.1) is satisfied and consider the abstract optimization problem (4.2). An element $\bar{x} \in S$ is called a $D_{\varphi}$-minimal (resp. weakly $D_{\varphi}$-minimal) solution of problem $(4.2)$ if $f(\bar{x})$ is a $D_{\varphi}$-minimal (resp. weakly $D_{\varphi}$-minimal) element of the image set $f(S)$.

To establish a necessary condition for weakly $D_{\varphi}$-minimal solutions of problem (4.2) we are first concerned with contingent cones. 
Theorem 4.2 Let $\left(X,\|\cdot\|_{X}\right)$ be a real normed space and $S \subset X, S \neq \emptyset$. Moreover, let $(Y,\langle\cdot, \cdot\rangle)$ be the given real Hilbert space and assume that $K \subset Y$ such that $K$ is convex and $\operatorname{int}(K) \neq \emptyset$. Let a map $r: X \rightarrow Y$ be given. If $r$ is Fr? chet differentiable at an $\bar{x} \in S$ with $r(\bar{x}) \in-K$, then

$$
\left\{h \in \mathcal{T}(S, \bar{x}): r(\bar{x})+r^{\prime}(\bar{x})(h) \in-\operatorname{int}(K)\right\} \subset \mathcal{T}(\{x \in S: r(x) \in-\operatorname{int}(K)\}, \bar{x}) .
$$

To prove this theorem we need a known auxiliary result (see [18], p. 7).

Lemma 4.3 Let $X$ be a real topological linear space and $S \subset X$ such that $S$ is convex and $\operatorname{int}(S) \neq \emptyset$. If $x \in S$ and $y \in \operatorname{int}(S)$, then

$$
\lambda x+(1-\lambda) y \in \operatorname{int}(S) \text { for all } \lambda \in[0,1) .
$$

Proof of Theorem 4.2: In the first part we follow the lines in [9], pp. 163. In the second part we complete the proof by applying Lemma 4.3 (we need this auxiliary result, because $K$ fails to be a cone in general; hence, some arguments used in [9] cannot be applied). Let an arbitrary $h \in \mathcal{T}(S, \bar{x})$ be given such that

$$
r(\bar{x})+r^{\prime}(\bar{x})(h) \in-\operatorname{int}(K) .
$$

If $h=0_{X}$, the statement trivially follows. Hence, let $h \neq 0_{X}$. Then, there are a sequence $\left(x_{n}\right)_{n \in \mathbb{N}}$ of elements $x_{n} \in S$ and a sequence $\left(\lambda_{n}\right)_{n \in \mathbb{N}}$ of positive real numbers such that $\bar{x}=\lim _{n \rightarrow \infty} x_{n}$ and $h=\lim _{n \rightarrow \infty} \lambda_{n}\left(x_{n}-\bar{x}\right)$. Since $h \neq 0_{X}$, it is easily seen that $\lim _{n \rightarrow \infty} \lambda_{n}=\infty$ and there is an $N \in \mathbb{N}$ such that $x_{n}-\bar{x} \neq 0_{X}$ for all $n \geq N$. Now defining $h_{n}:=\lambda_{n}\left(x_{n}-\bar{x}\right), n \in \mathbb{N}$, we obtain

$$
\begin{aligned}
r\left(x_{n}\right)= & \frac{1}{\lambda_{n}}\left(\lambda_{n}\left(r\left(x_{n}\right)-r(\bar{x})-r^{\prime}(\bar{x})\left(x_{n}-\bar{x}\right)\right)+r^{\prime}(\bar{x})\left(h_{n}-h\right)\right. \\
& \left.+r(\bar{x})+r^{\prime}(\bar{x})(h)\right)+\left(1-\frac{1}{\lambda_{n}}\right) r(\bar{x}) \text { for all } n \in \mathbb{N} .
\end{aligned}
$$

Next we verify the identity

$$
\lim _{n \rightarrow \infty}\left[\lambda_{n}\left(r\left(x_{n}\right)-r(\bar{x})-r^{\prime}(\bar{x})\left(x_{n}-\bar{x}\right)\right)+r^{\prime}(\bar{x})\left(h_{n}-h\right)\right]=0_{Y} .
$$

Since by assumption $r$ is Fr?chet differentiable at $\bar{x}$, using the above arguments we obtain

$$
\begin{aligned}
& \lim _{n \rightarrow \infty}\left[\lambda_{n}\left(r\left(x_{n}\right)-r(\bar{x})-r^{\prime}(\bar{x})\left(x_{n}-\bar{x}\right)\right)\right] \\
& =\lim _{n \rightarrow \infty} \lambda_{n}\left[r\left(\bar{x}+\left(x_{n}-\bar{x}\right)\right)-r(\bar{x})-r^{\prime}(\bar{x})\left(x_{n}-\bar{x}\right)\right] \\
& =\lim _{n \rightarrow \infty}\left\|h_{n}\right\|_{X} \frac{r\left(\bar{x}+\left(x_{n}-\bar{x}\right)\right)-r(\bar{x})-r^{\prime}(\bar{x})\left(x_{n}-\bar{x}\right)}{\left\|x_{n}-\bar{x}\right\|_{X}} \\
& =\lim _{n \rightarrow \infty}\left\|h_{n}\right\|_{X} \lim _{n \rightarrow \infty} \frac{r\left(\bar{x}+\left(x_{n}-\bar{x}\right)\right)-r(\bar{x})-r^{\prime}(\bar{x})\left(x_{n}-\bar{x}\right)}{\left\|x_{n}-\bar{x}\right\|_{X}}=0_{Y} .
\end{aligned}
$$


This together with

$$
\lim _{n \rightarrow \infty} r^{\prime}(\bar{x})\left(h_{n}-h\right)=r^{\prime}(\bar{x})\left(0_{X}\right)=0_{Y}
$$

implies (4.5). By (4.3) and (4.5) there is an $N_{1} \in \mathbb{N}$ such that

$$
\begin{aligned}
y_{n}:= & {\left[\lambda_{n}\left(r\left(x_{n}\right)-r(\bar{x})-r^{\prime}(\bar{x})\left(x_{n}-\bar{x}\right)\right)+r^{\prime}(\bar{x})\left(h_{n}-h\right)\right] } \\
& +r(\bar{x})+r^{\prime}(\bar{x})(h) \in-\operatorname{int}(K) \text { for all } n \geq N_{1} .
\end{aligned}
$$

Moreover, since $\lim _{n \rightarrow \infty} \lambda_{n}=\infty$, there is an $N_{2} \in \mathbb{N}$ such that $\frac{1}{\lambda_{n}}<1$ for all $n \geq N_{2}$. Set $N:=\max \left\{N_{1}, N_{2}\right\}$. Since $r(\bar{x}) \in-K$, using (4.4), (4.6) and Lemma 4.3 we then conclude that

$$
-r\left(x_{n}\right)=\frac{1}{\lambda_{n}}\left(-y_{n}\right)+\left(1-\frac{1}{\lambda_{n}}\right)(-r(\bar{x})) \in \operatorname{int}(K) \text { for all } n \geq N,
$$

i.e., $r\left(x_{n}\right) \in-\operatorname{int}(K)$ for all $n \geq N$. But this implies that

$$
h \in \mathcal{T}(\{x \in S: r(x) \in-\operatorname{int}(K)\}, \bar{x})
$$

which completes the proof of Theorem 4.2.

Now using Theorem 4.2 and the well-known Theorem of Lyusternik (see e.g. [10], [11], [19] or [8], pp. 96) we can establish a necessary condition for a weakly $D_{\varphi}$-minimal solution of problem (4.2).

Theorem 4.4 Let the abstract optimization problem (4.2) be given such that (4.1) is satisfied and assume that $\bar{x} \in S$ is a weakly $D_{\varphi}$-minimal solution of (4.2). Moreover, let $f$ and $g$ be Fr? chet differentiable at $\bar{x}$, let $h$ be continuously Fr? chet differentiable at $\bar{x}$ and $h^{\prime}(\bar{x})$ be surjective. Then, there is no $x \in \operatorname{int}(\hat{S})$ such that

$$
f^{\prime}(\bar{x})(x-\bar{x}) \in-\operatorname{int}\left(D_{\varphi}\right), \quad g(\bar{x})+g^{\prime}(\bar{x})(x-\bar{x}) \in-\operatorname{int}\left(C_{Z_{1}}\right)
$$

and

$$
h^{\prime}(\bar{x})(x-\bar{x})=0_{Z_{2}} .
$$

Proof: On the contrary, assume that there is an $x \in \operatorname{int}(\hat{S})$ such that $f^{\prime}(\bar{x})(x-\bar{x}) \in-\operatorname{int}\left(D_{\varphi}\right), g(\bar{x})+g^{\prime}(\bar{x})(x-\bar{x}) \in-\operatorname{int}\left(C_{Z_{1}}\right)$ and $h^{\prime}(\bar{x})(x-\bar{x})=0_{Z_{2}}$. We distinguish two cases.

First case. Assume that $x=\bar{x}$. This implies $0_{Y}=f^{\prime}(\bar{x})\left(0_{X}\right)=f^{\prime}(\bar{x})(x-$ $\bar{x}) \in-\operatorname{int}\left(D_{\varphi}\right)$ resp. $0_{Y} \in \operatorname{int}\left(D_{\varphi}\right)$, contradicting (4.1) where we have assumed that $D_{\varphi} \subset H^{+}$.

Second case. Assume that $x \neq \bar{x}$. Since Theorem 4.2 and also the Theorem of Lyusternik are available, this case can be treated analogously to a more special case in [9], pp. 165.

We are now in position to formulate the announced generalization of the Lagrange multiplier rule. For verifying the following result the separation theorem of Eidelheit (see [9], p. 74) and Theorem 4.4 play an essential role. 
Theorem 4.5 Let the abstract optimization problem (4.2) be given such that (4.1) is satisfied and assume that $\bar{x} \in S$ is a weakly $D_{\varphi}$-minimal solution of (4.2). Moreover, let $f$ and $g$ be Fr? chet differentiable at $\bar{x}$, let $h$ be continuously Fr? chet differentiable at $\bar{x}$ and let the image set $h^{\prime}(\bar{x})(X)$ be closed. Then, there are a real number $\lambda \geq 0$ and continuous linear functionals $u \in C_{Z_{1}}^{*}$ and $v \in Z_{2}^{*}$ with $(\lambda, u, v) \neq\left(0,0_{Z_{1}^{*}}, 0_{Z_{2}^{*}}\right)$ such that

$$
\lambda\left\langle a, f^{\prime}(\bar{x})(x-\bar{x})\right\rangle+\left(u \circ g^{\prime}(\bar{x})+v \circ h^{\prime}(\bar{x})\right)(x-\bar{x}) \geq 0 \text { for all } x \in \hat{S}
$$

and

$$
(u \circ g)(\bar{x})=0 .
$$

If in addition, there is an element $\hat{x} \in \operatorname{int}(\hat{S})$ such that $g(\bar{x})+g^{\prime}(\bar{x})(\hat{x}-\bar{x}) \in$ $-\operatorname{int}\left(C_{Z_{1}}\right)$ and $h^{\prime}(\bar{x})(\hat{x}-\bar{x})=0_{Z_{2}}$, and if the map $h^{\prime}(\bar{x})$ is surjective, then $\lambda>0$.

To verify Theorem 4.5 we need an auxiliary result which is a direct consequence of Theorem 2.102 in [12].

Lemma 4.6 Let $D_{\varphi}^{*}$ be the dual set of $D_{\varphi}$. If the property $D_{\varphi} \subset H^{+}$is satisfied, then $D_{\varphi}^{*}=\operatorname{cone}(\{a\})$.

Proof of Theorem 4.5: Using the same kind of arguments as in [9], pp. 166 we conclude that there are continuous linear functionals $t \in D_{\varphi}^{*}, u \in C_{Z_{1}}^{*}$ and $v \in Z_{2}^{*}$ such that $(t, u, v) \neq 0_{Y^{*} \times Z_{1}^{*} \times Z_{2}^{*}}$, $(u \circ g)(\bar{x})=0$ and

$$
\left(t \circ f^{\prime}(\bar{x})+u \circ g^{\prime}(\bar{x})+v \circ h^{\prime}(\bar{x})\right)(x-\bar{x}) \geq 0 \text { for all } x \in \hat{S} .
$$

Moreover, since $D_{\varphi} \subset H^{+}$, the equality $D_{\varphi}^{*}=\operatorname{cone}(\{a\})$ follows from Lemma 4.6. Hence, there is a $\lambda \geq 0$ with $t=\lambda a$. Then, the assertion (4.7) follows directly from (4.9).

If in addition, there is an element $\hat{x} \in \operatorname{int}(\hat{S})$ such that $g(\bar{x})+g^{\prime}(\bar{x})(\hat{x}-\bar{x}) \in$ $-\operatorname{int}\left(C_{Z_{1}}\right)$ and $h^{\prime}(\bar{x})(\hat{x}-\bar{x})=0_{Z_{2}}$, and if the map $h^{\prime}(\bar{x})$ is surjective, then the property $t \neq 0_{Y^{*}}$ follows from arguments used in [9], p. 168. This implies $\lambda>0$.

Remark 4.7 (i) The necessary optimality conditions presented in Theorem 4.5 generalize not only the Lagrange multiplier rule, but also the Fritz-Johnconditions. Moreover, if $\lambda$ is positive (for instance, if the constraint qualification in the second part of Theorem 4.5 is satisfied), then we even obtain an extension of the Karush-Kuhn-Tucker-conditions (compare e.g. [9], p. 168).

(ii) If $\hat{S}=X$, then inequality (4.7) simplifies to

$$
(\lambda a) \circ f^{\prime}(\bar{x})+u \circ g^{\prime}(\bar{x})+v \circ h^{\prime}(\bar{x})=0_{X^{*}} .
$$


We now apply the generalized multiplier rule stated in Theorem 4.5 to the case of finite-dimensional Hilbert spaces $Y$. Let us first consider a multiobjective optimization problem, i.e., we are concerned with problem (4.2) in the situation $Y=\mathbb{R}^{n}$.

Theorem 4.8 Let $Y=\mathbb{R}^{n}$ and assume that $f: \mathbb{R}^{m} \rightarrow \mathbb{R}^{n}, g: \mathbb{R}^{m} \rightarrow \mathbb{R}^{k}$ and $h: \mathbb{R}^{m} \rightarrow \mathbb{R}^{p}$ are given maps. Let $\mathbb{R}^{k}$ be partially ordered by the natural ordering cone $\mathbb{R}_{+}^{k}$. Assume that $D_{\varphi}$ is convex and satisfies $D_{\varphi} \subset H^{+}$. Let the feasible set $S$ be defined by

$S:=\left\{x \in \mathbb{R}^{m}: g_{i}(x) \leq 0\right.$ for all $i \in\{1, \ldots, k\}, h_{i}(x)=0$ for all $\left.i \in\{1, \ldots, p\}\right\}$.

Moreover, let $\bar{x} \in S$ be a weakly $D_{\varphi}$-minimal solution of the multiobjective optimization problem

$$
\min _{x \in S} f(x)
$$

and assume that $f$ and $g$ are differentiable at $\bar{x}$ and that $h$ is continuously differentiable at $\bar{x}$. Let an $x \in \mathbb{R}^{m}$ be given such that

$\nabla g_{i}(\bar{x})^{T}(x-\bar{x})<0$ for all $i \in I(\bar{x}), \quad \nabla h_{i}(\bar{x})^{T}(x-\bar{x})=0$ for all $i \in\{1, \ldots, p\}$

where the index set $I(\bar{x})$ is defined by

$$
I(\bar{x}):=\left\{i \in\{1, \ldots, k\}: g_{i}(\bar{x})=0\right\}
$$

(the set of the active inequality restrictions at $\bar{x}$ ). Finally, assume that the gradients $\nabla h_{1}(\bar{x}), \ldots, \nabla h_{p}(\bar{x})$ are linearly independent. Then, there are multipliers $u_{i} \geq 0(i \in I(\bar{x}))$ and $v_{i} \in \mathbb{R}(i \in\{1, \ldots, p\})$ with the property

$$
\sum_{i=1}^{n} a_{i} \nabla f_{i}(\bar{x})+\sum_{i \in I(\bar{x})} u_{i} \nabla g_{i}(\bar{x})+\sum_{i=1}^{p} v_{i} \nabla h_{i}(\bar{x})=0 \mathbb{R}^{m}
$$

Proof: Applying Theorem 4.5 and Remark 4.7 we can similarly argue as in [9], pp. 173 where a more special case has been considered.

We are now interested in the case when $Y=S^{n}$ where $S^{n}$ denotes the vector space of all real symmetric $(n \times n)$ - matrices. Let this finite-dimensional space be endowed with the inner product $\langle\cdot, \cdot\rangle$ which is defined by

$$
\left\langle M_{1}, M_{2}\right\rangle:=\operatorname{trace}\left(M_{1} \cdot M_{2}\right) \text { for all } M_{1}, M_{2} \in S^{n} .
$$

Theorem 4.9 Let $Y=S^{n}$ and assume that $F: \mathbb{R}^{m} \rightarrow S^{n}$ and $G: \mathbb{R}^{m} \rightarrow$ $S^{k}$ are given matrix valued maps. Let the space $S^{k}$ be partially ordered by a convex cone $C \subset S^{k}$. Assume that $D_{\varphi}$ is convex and satisfies $D_{\varphi} \subset H^{+}$. Let the feasible set $S$ be defined by

$$
S:=\left\{x \in \mathbb{R}^{m}: G(x) \in-C\right\} .
$$


Moreover, let $\bar{x} \in S$ be a weakly $D_{\varphi}$-minimal solution of the conic optimization problem

$$
\min _{x \in S} F(x)
$$

and assume that $F$ and $G$ are elementwise differentiable at $\bar{x}$. Then, there are a real number $\lambda \geq 0$ and a matrix $L \in C^{*}$ with $(\lambda, L) \neq\left(0,0_{S^{k}}\right)$ such that

$$
(\lambda a) \circ F^{\prime}(\bar{x})+L \circ G^{\prime}(\bar{x})=0_{\mathbb{R}^{m}}
$$

and

$$
\langle L, G(\bar{x})\rangle=0 .
$$

If in addition, the equality

$$
G^{\prime}(\bar{x})\left(\mathbb{R}^{m}\right)+\operatorname{cone}(C+\{G(\bar{x})\})=S^{k}
$$

holds, then $\lambda>0$.

Proof: Applying Theorem 4.5 and Remark 4.7 we can similarly argue as in [8], pp. 204 where a more special case has been considered.

Remark 4.10 The vector space $S^{k}$ considered in Theorem 4.9 is partially ordered by a convex cone $C \subset S^{k}$. Typical examples for such convex cones can be found in [8], pp. 189, for instance the L?wner cone

$$
S_{+}^{k}:=\left\{M \in S^{k}: M \text { is positive semidefinite }\right\},
$$

the $K$-copositive ordering cone (where $K \subset \mathbb{R}^{k}$ is a given convex cone)

$$
C_{K}^{k}:=\left\{M \in S^{k}: x^{T} M x \geq 0 \text { for all } x \in K\right\},
$$

the nonnegative ordering cone

$$
N^{k}:=\left\{M \in S^{k}: m_{i j} \geq 0 \text { for all } i, j \in\{1, \ldots, k\}\right\}
$$

and the double nonnegative ordering cone

$$
D^{k}:=S_{+}^{k} \cap N^{k} .
$$

Remark 4.11 (i) It would be of interest under what additional hypotheses the necessary conditions given in the Theorems 4.8 and 4.9 are also sufficient. Typically, certain generalized convexity concepts like quasiconvexity or pseudoconvexity are needed to adapt them at the reference set $D_{\varphi}$.

(ii) Another subject not having studied in our paper would be a duality theory for optimization problems which are based on the relation $\preceq_{\varphi}$. 


\section{References}

[1] J.M. Borwein, Proper efficient points for maximizations with respect to cones, SIAM J. Control Optim., 15 (1977), 57 - 63.

[2] J.M. Borwein, The geometry of Pareto efficiency over cones, Math. Operationsforsch. Statist. Ser. Optim., 11 (1980), 235 - 248.

[3] C. Gerstewitz (Tammer), Nichtkonvexe Dualität in der Vektoroptimierung, Wissensch. Zeitschr. TH Leuna-Merseburg, 25 (1983), 357 364 .

[4] C. Gerstewitz (Tammer), E. Iwanow, Dualität für nichtkonvexe Vektoroptimierungsprobleme, Wissensch. Zeitschr. TH Ilmenau, 31 (1985), 61 81.

[5] C. Gerth (Tammer), P. Weidner, Nonconvex separation theorems and some applications in vector optimization, J. Optim. Theory Appl., 67 (1990), $297-320$.

[6] J. Jahn, Scalarization in vector optimization, Math. Program., 29 (1984), $203-218$.

[7] J. Jahn, Existence theorems in vector optimization, J. Optim. Theory Appl., 50 (1986) 397-406.

[8] J. Jahn, Introduction to the Theory of Nonlinear Optimization, Springer, Berlin, 2007.

[9] J. Jahn, Vector Optimization - Theory, Applications, and Extensions, Springer, Berlin, 2011.

[10] A. Kirsch, W. Warth and J. Werner, Notwendige Optimalitätsbedingungen und ihre Anwendung, Lecture Notes in Economics and Mathematical Systems, 152, Springer, Berlin, 1978.

[11] L.A. Lyusternik, W.I. Sobolew, Elemente der Funktionalanalysis, Akademie-Verlag, Berlin, 1968.

[12] C. Sommer, Eine neue Ordnungsrelation in der Vektoroptimierung, Dissertation, Universität Erlangen-Nürnberg, http://www.mso.math.fau.de/sommer, 2012.

[13] C. Sommer, A new parameterized binary relation in vector optimization, Preprint, Erlangen, 2012. 
[14] W. Vogel, Vektoroptimierung in Produkträumen, Hain, Meisenheim am Glan, 1977.

[15] P. Weidner, Dominanzmengen und Optimalitätsbegriffe in der Vektoroptimierung, Wissensch. Zeitschr. TH Ilmenau, 31 (1985), 133 - 146.

[16] P. Weidner, Charakterisierung von Mengen effizienter Elemente in linearen Räumen auf der Grundlage allgemeiner Bezugsmengen, Dissertation (A), Universität Halle-Wittenberg, 1985.

[17] P. Weidner, Comparison of six types of separating functionals, in: H.J. Sebastian, K. Tammer (eds.): System modelling and optimization, Leipzig, 1989, Springer, Berlin, 1990.

[18] P. Weidner, Ein Trennungskonzept und seine Anwendungen auf Vektoroptimierungsverfahren, Dissertation (B), Universität Halle-Wittenberg, 1990.

[19] J. Werner, Optimization - Theory and applications, Vieweg, Braunschweig, 1984.

Received: February 10, 2013 